\title{
ON A PROBLEM OF LOWER LIMIT IN THE STUDY OF NONRESONANCE
}

\author{
A. ANANE AND O. CHAKRONE
}

AbStract. We prove the solvability of the Dirichlet problem

$$
\left\{\begin{aligned}
-\Delta_{p} u & =f(u)+h & & \text { in } \Omega, \\
u & =0 & & \text { on } \partial \Omega
\end{aligned}\right.
$$

for every given $h$, under a condition involving only the asymptotic behaviour of the potential $F$ of $f$ with respect to the first eigenvalue of the p-Laplacian $\Delta_{p}$. More general operators are also considered.

\section{INTRODUCTION}

This paper is concerned with the existence of solutions for the problem

$$
\left(\mathcal{P}_{p}\right)\left\{\begin{aligned}
-\Delta_{p} u & =f(u)+h & \text { in } & \Omega \\
u & =0 & & \text { on } \partial \Omega
\end{aligned}\right.
$$

where $\Omega$ is a bounded domain of $\mathbb{R}^{N}, N \geq 1, \Delta_{p}$ denotes the p-Laplacian $\Delta_{p} u=\operatorname{div}\left(|\nabla u|^{p-2} \nabla u\right), 1<p<\infty, f$ is a continuous function from $\mathbb{R}$ to $\mathbb{R}$ and $h$ is a given function on $\Omega$.

A classical result, essentially due to Hammerstein $[\mathrm{H}]$, asserts that if $f$ satisfies a suitable polynomial growth restriction connected with the Sobolev imbeddings and if

$$
\limsup _{s \rightarrow \pm \infty} \frac{2 F(s)}{|s|^{2}}<\lambda_{1},
$$

then problem $\left(\mathcal{P}_{2}\right)$ is solvable for any $h$. Here $F$ denotes the primitive $F(s)=\int_{0}^{s} f(t) d t$ and $\lambda_{1}$ is the first eigenvalue of $-\Delta$ on $H_{0}^{1}(\Omega)$. Several improvements of this result have been considered in the recent years.

1991 Mathematics Subject Classification. 35J60, 35P30.

Key words and phrases. p-Laplacian, nonresonance, first eigenvalue.

Received: May 20, 1997. 
In 1989 , the case $\mathrm{N}=1$ and $\mathrm{p}=2$ was considered in $[\mathrm{Fe}, \mathrm{O}, \mathrm{Z}]$. It was shown there that $\left(\mathcal{P}_{2}\right)$ is solvable for any $h \in L^{\infty}(\Omega)$ if

$$
\liminf _{s \rightarrow \pm \infty} \frac{2 F(s)}{|s|^{2}}<\lambda_{1}
$$

If $N \geq 1$ and $\mathrm{p}=2,[\mathrm{~F}, \mathrm{G}, \mathrm{Z}]$ showed later that $\left(\mathcal{P}_{2}\right)$ is solvable for any $h \in$ $L^{\infty}(\Omega)$ if

$$
\liminf _{s \rightarrow \pm \infty} \frac{2 F(s)}{|s|^{2}}<\left(\frac{\pi}{2 R(\Omega)}\right)^{2},
$$

where $R(\Omega)$ denotes the radius of the smallest open ball $B(\Omega)$ containing $\Omega$. This result was extended to the p-laplacian case in [E,G.1], where solvability of $\left(\mathcal{P}_{p}\right)$ was derived under the condition

$$
\liminf _{s \rightarrow \pm \infty} \frac{p F(s)}{|s|^{p}}<(p-1)\left\{\frac{1}{R(\Omega)} \int_{0}^{1} \frac{d t}{\left(1-t^{p}\right)^{\frac{1}{p}}}\right\}^{p} .
$$

Note that $\left(F_{4}\right)$ reducer to $\left(F_{3}\right)$ when $p=2$.

The question now naturally arises whether $(p-1)\left\{\frac{1}{R(\Omega)} \int_{0}^{1} \frac{d t}{\left(1-t^{p}\right)^{\frac{1}{p}}}\right\}^{p}$ can be replaced by $\lambda_{1}$ in $\left(F_{4}\right)$, where $\lambda_{1}$ denotes the first eigenvalue of $-\Delta_{p}$ on $W_{0}^{1, p}(\Omega)$ (cf. Anane $\left.[\mathrm{A}]\right)$.

Observe that for $N>1$ and $p=2,\left(\frac{\pi}{2 R(\Omega)}\right)^{2}<\lambda_{1}$, and a similar strict inequality holds when $1<p<\infty$. One of our purposes in this paper is to show that the constants in $\left(F_{3}\right)$ and $\left(F_{4}\right)$ can be inproved a little bit.

Denote by $l(\Omega)=l$ the length of the smallest edge of an arbitrary parallelepiped containing $\Omega$. In the first part of the paper we assume

$$
\liminf _{s \rightarrow \pm \infty} \frac{p F(s)}{|s|^{p}}<C_{p}(l)
$$

where $C_{p}(l)=C_{p}=(p-1)\left\{\frac{2}{l} \int_{0}^{1} \frac{d t}{\left(1-t^{p}\right)^{\frac{1}{p}}}\right\}^{p}$.

Observe that for $N=1, C_{p}=\lambda_{1}$ is the first eigenvalue of $-\Delta_{p}$ on $\left.\Omega=\right] 0, l[$. In particular: $C_{2}=\left(\frac{\pi}{l}\right)^{2}$, and we recover the result of [ Fe,O,Z]. It is clear that $\left(F_{5}\right)$ is a weaker hypothesis than $\left(F_{4}\right)$. The difference between $\left(F_{5}\right)$ and $\left(F_{4}\right)$ is particularly important when $\Omega$ is a rectangle or a triangle. However $C_{p}(l)<\lambda_{1}$ when $N>1$, and the question raised above remains open.

In the second part of the paper we investigate the question of replacing $\Delta_{p}$ by the second order elliptic operator

$$
A_{p}(u)=\sum_{1 \leq i, j \leq N} \frac{\partial}{\partial x_{i}}\left(|\nabla u|_{a}^{p-2} a_{i j}(x) \frac{\partial u}{\partial x_{j}}\right),
$$

where $|\nabla u|_{a}^{2}=\sum_{1 \leq i, j \leq N} a_{i j}(x) \frac{\partial u}{\partial x_{i}} \frac{\partial u}{\partial x_{j}}$. Observe that the method used in $[F, G, Z]$, and [E,G.1] exploits the symmetry of the Laplacian or p-laplacian. It is not clear whether it can be adapted to more general second order elliptic operators like $A_{p}$ above.

While this paper was being completed, we learned of a work by P.Omari and Grossinho (Cf. [GR,O.1], [GR,O.2]), where a result of the same type as 
ours is established in the case of the linear operator $A_{2}(u)$. The authors in [GR,O.2] also consider parabolic operators.

\section{The CASE OF THE $p$-LAPLACIAN}

In this section we will consider the problem $\left(\mathcal{P}_{p}\right)$ where $\Omega$ is a bounded domain of $\mathbb{R}^{N}, N \geq 1,1<p<\infty, f$ is a continuous function from $\mathbb{R}$ to $\mathbb{R}$ and $h \in L^{\infty}(\Omega)$.

Denote by $[A B]$ the smallest edge of an arbitrary parallelepiped containing $\Omega$. Making an orthogonal change of variables, we can always suppose that $A B$ is parallel to one of the axis of $\mathbb{R}^{N}$. So $\Omega \subset P=\prod_{j=1}^{N}\left[a_{j}, b_{j}\right]$ with, for some $i,|A B|=b_{i}-a_{i}=\min _{1 \leq j \leq N}\left\{b_{j}-a_{j}\right\}$, a quantity which we denote by $l(\Omega)=l$.

Theorem 1. Assume

$$
\liminf _{s \rightarrow \pm \infty} \frac{p F(s)}{|s|^{p}}<C_{p}
$$

where $C_{p}=C_{p}(l)$ is defined in the introduction. Then for any $h \in L^{\infty}(\Omega)$ $\left(\mathcal{P}_{p}\right)$ has a solution $u \in W_{0}^{1, p}(\Omega) \cap C^{1}(\Omega)$.

Definition 1. An upper solution for $\left(\mathcal{P}_{p}\right)$ is defined as a function $\beta: \bar{\Omega} \rightarrow \mathbb{R}$ such that:

- $\beta \in C^{1}(\bar{\Omega})$

- $\Delta_{p} \beta \in C(\bar{\Omega})$

- $-\Delta_{p} \beta(x) \geq f(\beta(x))+h(x)$ a.e.x in $\Omega$

A lower solution $\alpha$ is defined by reversing the inequalities above.

Lemma 1. Assume that $\left(\mathcal{P}_{p}\right)$ admits an upper solution $\beta$ and a lower solution $\alpha$ with $\alpha(x) \leq \beta(x)$ in $\Omega$. Then $\left(\mathcal{P}_{p}\right)$ admits a solution $u \in W_{0}^{1, p}(\Omega) \cap$ $C^{1}(\Omega)$, with $\alpha(x) \leq u(x) \leq \beta(x)$ in $\Omega$.

Proof. This lemma is well known when $p=2$ (see, e.g., [F.G.Z]). We sketch a proof in the general case $1<p<\infty$.

Define

$$
\tilde{f}(x, s)=\left\{\begin{array}{cll}
f(\beta(x)) & \text { if } & s \geq \beta(x) \\
f(s) & \text { if } & \alpha(x) \leq s \leq \beta(x) \\
f(\alpha(x)) & \text { if } & s \leq \alpha(x) .
\end{array}\right.
$$

By a simple fixed point argument and the results of Di Benedetto [B], there is a solution $u \in W_{0}^{1, p}(\Omega) \cap C^{1}(\Omega)$ of

$$
(\tilde{\mathcal{P}})\left\{\begin{aligned}
-\Delta_{p} u & =\tilde{f}(x, u)+h(x) \text { in } \Omega, \\
u & =0 \text { on } \partial \Omega .
\end{aligned}\right.
$$

We claim that $\alpha(x) \leq u(x) \leq \beta(x)$ in $\Omega$, which clearly implies the conclusion. To prove the first inequality, one multiplies the equation $(\tilde{\mathcal{P}})$ by $w=u-u_{\alpha}$, where $u_{\alpha}(x)=\max (u(x), \alpha(x))$, integrates by parts and uses the fact that $\alpha$ is a lower solution we obtain $\left\langle\left(-\Delta_{p} u\right)-\left(-\Delta_{p}(u-w)\right), w\right\rangle \leq 0$, which implies $w=0$ (since $-\Delta_{p}$ is strictly monotone). 
Lemma 2. Let $a<b$ and $M>0$, and assume

$$
\liminf _{s \rightarrow+\infty} \frac{p F(s)}{|s|^{p}}<C_{p}(b-a) .
$$

Then there exists $\beta_{1} \in C^{1}(I)$ such that $\Delta_{p} \beta_{1} \in C(I)$ and

$$
\left\{\begin{aligned}
-\Delta_{p} \beta_{1}(t) & \geq f\left(\beta_{1}(t)\right)+M & \forall t \in I, \\
\beta_{1}(t) & \geq 0 & \forall t \in I,
\end{aligned}\right.
$$

where $I=[a, b]$

Lemma 3. Assume

$$
\liminf _{s \rightarrow-\infty} \frac{p F(s)}{|s|^{p}}<C_{p}(b-a) .
$$

Then there exists $\alpha_{1} \in C^{1}(I)$ such that $\Delta_{p} \alpha_{1} \in C(I)$ and

$$
\left\{\begin{aligned}
-\Delta_{p} \alpha_{1}(t) & \leq f\left(\alpha_{1}(t)\right)-M & \forall t \in I, \\
\alpha_{1}(t) & \leq 0 & \forall t \in I .
\end{aligned}\right.
$$

Accepting for a moment the conclusion of these two lemmas, let us turn to the

Proof of Theorem 1. By Lemma 1 it suffices to show the existence of an upper solution and a lower solution for $\left(\mathcal{P}_{p}\right)$. Let us describe the construction of the upper solution (that of the lower solution is similar).

Let $M>\|h\|_{\infty}$ and $i \in\{1,2, \ldots, N\}$ such that $b-a=b_{i}-a_{i}=\min _{1 \leq j \leq N} b_{j}-a_{j}$.

By Lemma 2 there exists $\beta_{1}: I \rightarrow \mathbb{R}$ such that $\beta_{1} \in C^{1}(I), \Delta_{p} \beta_{1} \in C(I)$ and

$$
\left\{\begin{aligned}
-\Delta_{p} \beta_{1}(t) & \geq f\left(\beta_{1}(t)\right)+M & \forall t \in I \\
\beta_{1}(t) & \geq 0 & \forall t \in I .
\end{aligned}\right.
$$

Writing $\beta(x)=\beta_{1}\left(x_{i}\right)$ for all $x \in \bar{\Omega}$, it is clear that $\beta \in C^{1}(\bar{\Omega}),-\Delta_{p} \beta(x)=$ $-\Delta_{p} \beta_{1}\left(x_{i}\right) \in C(\bar{\Omega})$, and we have:

$$
\begin{aligned}
-\Delta_{p} \beta(x) & =-\Delta_{p} \beta_{1}\left(x_{i}\right) \\
& \geq f\left(\beta_{1}\left(x_{i}\right)\right)+M \\
& =f(\beta(x))+M \\
& \geq f(\beta(x))+h(x) \quad \text { a.e. } x \in \Omega .
\end{aligned}
$$

The proof of Theorem 1 is thus complete.

Proof of Lemma 2. The proof of Lemma 3 follows simiarly.

\section{First case.}

Suppose $\inf _{s \geq 0} f(s)=-\infty$. Then $\exists \beta \in \mathbb{R}_{+}^{*}$ such that $f(\beta)<-M$, and the constant function $\beta$ provides a solution to the problem in Lemma 2.

\section{Second case.}

Suppose now $\inf _{s \geq 0} f(s)>-\infty$. Let $K>M$ such that $\inf _{s \geq 0} f(s)>-K+1$.

Thus $f(s)+K \geq 1$ for all $s \geq 0$. Define $g: \mathbb{R} \rightarrow \mathbb{R}$ by:

$$
g(s)=\left\{\begin{array}{lll}
f(s)+K & \text { if } \quad s \geq 0 \\
f(0)+K & \text { if } \quad s<0
\end{array}\right.
$$


and denote $G(s)=\int_{0}^{s} g(t) d t$ for all $s$ in $\mathbb{R}$. It is easy to see that $g(s) \geq 1 \forall s \in \mathbb{R}$ and that

$$
\liminf _{s \rightarrow+\infty} \frac{p G(s)}{s^{p}}=\liminf _{s \rightarrow+\infty} \frac{p F(s)}{s^{p}}<C_{p} .
$$

Now it is clearly sufficient to prove the existence of a function $\beta_{1}: I \rightarrow \mathbb{R}$ such that $\beta_{1} \in C^{1}(I), \Delta_{p} \beta_{1} \in C(I)$ and

$$
\left\{\begin{array}{ccc}
-\Delta_{p} \beta_{1}(t) & =g\left(\beta_{1}(t)\right) & \forall t \in I, \\
\beta_{1}(t) & \geq 0 & \forall t \in I .
\end{array}\right.
$$

For that purpose we will need the following three lemmas.

Lemma 4. Define

$$
\tau_{G}(d)=\int_{0}^{d} \frac{d t}{(p\{G(d)-G(s)\})^{\frac{1}{p}}}
$$

for $d>0$. Then

$$
\limsup _{d \rightarrow+\infty} \tau_{G}(d) \geq\left(\int_{0}^{1} \frac{d t}{\left(1-t^{p}\right)^{\frac{1}{p}}}\right)\left(\liminf _{s \rightarrow+\infty} \frac{p G(s)}{|s|^{p}}\right)^{\frac{-1}{p}} .
$$

In particular $\left(F^{+}\right)$implies $\limsup _{d \rightarrow+\infty} \tau_{G}(d)>(p-1)^{-\frac{1}{p}} \frac{(b-a)}{2}$.

Proof. Let be a positive number such that $\liminf _{s \rightarrow+\infty} \frac{p G(s)}{s^{p}}<\rho<C_{p}$. Then $\lim \sup _{s \rightarrow+\infty}(K(s))=+\infty$ where $K(s)=\rho|s|^{p}-p G(s)$. Let $w_{n}$ be the smallest number in $[0, n]$ such that $\max _{0 \leq s \leq n} K(s)=K\left(w_{n}\right)$; it is easily seen that $w_{n}$ is increasing with respect to $n$. Since $\rho|s|^{p}-p G(s)<$ $\rho w_{n}^{p}-p G\left(w_{n}\right) \quad \forall s \in\left[0, w_{n}[\right.$, we have

$$
\begin{aligned}
\tau_{G}\left(w_{n}\right) & >\rho^{-\frac{1}{p}} \int_{0}^{w_{n}} \frac{d t}{\left(w_{n}^{p}-s^{p}\right)^{\frac{1}{p}}} \\
& =\rho^{-\frac{1}{p}} \int_{0}^{1} \frac{d t}{\left(1-s^{p}\right)^{\frac{1}{p}}}
\end{aligned}
$$

and therefore

$$
\limsup _{d \rightarrow+\infty} \tau_{G}(d) \geq \rho^{-\frac{1}{p}} \int_{0}^{1} \frac{d t}{\left(1-s^{p}\right)^{\frac{1}{p}}}
$$

for all $\rho$ such that $\liminf _{s \rightarrow+\infty} \frac{p G(s)}{s^{p}}<\rho<C_{p}$, which clearly implies the lemma.

Lemma 5. Let $d>0$ and consider the mapping $T_{d}$ defined by

$$
T_{d}(u)(t)=d-\int_{a}^{t}\left(\left[\int_{a}^{r} g(u(s)) d s\right]^{\frac{1}{p-1}}\right) d r
$$

in the Banach space $C(I)$. Then $T_{d}$ has a fixed point.

Proof. Clearly by Ascoli's theorem $T_{d}$ is compact. The proof of Lemma 5 uses a homotopy argument based on the Leray Schauder topological degree. So $T$ will have a fixed point if the following condition holds: $\exists r>0$ such that $\left(I-\lambda T_{d}\right)(u) \neq 0 \forall u \in \partial B(0, r) \forall \lambda \in[0,1]$, where $\partial B(0, r)=$ $\left\{u \in C(I) ;\|u\|_{\infty}=r\right\}$. 
To prove that this condition holds, suppose by contradiction that $\forall n=$ $1,2, \ldots \exists u_{n} \in \partial B(0, n), \exists \lambda_{n} \in[0,1]$ such that: $u_{n}=\lambda_{n} T_{d}\left(u_{n}\right)$. The latter relation means

$$
u_{n}=\lambda_{n} d-\lambda_{n} \int_{a}^{t}\left(\left\{\int_{a}^{r} g\left(u_{n}(s)\right) d s\right\}^{\frac{1}{p-1}}\right) d r .
$$

Therefore $u_{n} \in C^{1}(I)$ and we have successively

$$
\left\{\begin{array}{l}
u_{n}^{\prime}(t)=-\lambda_{n}\left\{\int_{a}^{t} g\left(u_{n}(s)\right) d s\right\}^{\frac{1}{p-1}} \leq 0 \\
u_{n}^{\prime}(a)=0
\end{array}\right.
$$

$\Delta_{p} u_{n} \in C(I)$ and

$$
\begin{aligned}
-\Delta_{p} u_{n}(t) & =-\left(\left|u_{n}^{\prime}(t)\right|^{p-2} u_{n}^{\prime}(t)\right)^{\prime} \\
& =\left(\left(-u_{n}^{\prime}(t)\right)^{p-1}\right)^{\prime} \\
& =\lambda_{n}^{p-1} g\left(u_{n}(t)\right) .
\end{aligned}
$$

Note that by $(2), u_{n}^{\prime}(t)<0$ in $\left.] a, b\right]$, so that $u_{n}$ is decreasing.

Hence, for $n>d, u_{n}(b)=-n$. Multiplying the equation $(3)$ by $u_{n}^{\prime}(t)$, we obtain

$$
-\frac{p-1}{p} \frac{d}{d t}\left(-u_{n}^{\prime}(t)\right)^{p}=\lambda_{n}^{p-1} \frac{d}{d t} G\left(u_{n}(t)\right) .
$$

Indeed

$$
\begin{aligned}
\left(\left(-u_{n}^{\prime}(t)\right)^{p}\right)^{\prime} & =\left(\left(\left(-u_{n}^{\prime}(t)\right)^{p-1}\right)^{\frac{p}{p-1}}\right)^{\prime} \\
& =\frac{p}{p-1}\left(\left(-u_{n}^{\prime}(t)\right)^{p-1}\right)^{\frac{p}{p-1}}-1 \\
& =-\frac{p}{p-1} u_{n}^{\prime}(t)\left(\left(-u_{n}^{\prime}(t)\right)^{p-1}\right)^{\prime} \\
&
\end{aligned}
$$

By (4) we have

$$
\begin{aligned}
(p-1)\left(-u_{n}^{\prime}(t)\right)^{p} & =\lambda_{n}^{p-1} p\left[G\left(\lambda_{n} d\right)-G\left(u_{n}(t)\right)\right] \\
& \leq p\left[G(d)-G\left(u_{n}(t)\right)\right]
\end{aligned}
$$

since $G$ is increasing. Hence $(p-1)^{\frac{1}{p}}\left(-u_{n}^{\prime}(t)\right)\left\{p\left[G(d)-G\left(u_{n}(t)\right)\right]\right\}^{-\frac{1}{p}} \leq 1$. Integrating from $a$ to $b$ and changing variable $s=u_{n}(t) \quad\left(u_{n}(a)=\lambda_{n} d\right.$ and $\left.u_{n}(b)=-n\right)$, we obtain

$$
(p-1)^{\frac{1}{p}} \int_{-n}^{\lambda_{n} d}[p(G(d)-G(s))]^{-\frac{1}{p}} d s \leq b-a,
$$

i.e.

$$
\begin{aligned}
0 & \leq(p-1)^{\frac{1}{p}} \int_{0}^{\lambda_{n} d}[p(G(d)-G(s))]^{-\frac{1}{p}} d s \\
& =(b-a)+(p-1)^{\frac{1}{p}} \int_{0}^{-n}[p(G(d)-G(s))]^{-\frac{1}{p}} d s .
\end{aligned}
$$

Since $G(s)=s g(0)$ for $s \leq 0$, we obtain

$$
\begin{aligned}
0 & \leq(b-a)+(p-1)^{\frac{1}{p}} \int_{0}^{-n}[p(G(d)-s g(0))]^{-\frac{1}{p}} d s \\
& =(b-a)-\frac{(p-1)^{\frac{1}{p}}}{(p-1) g(0)}[p(G(d)+n g(0))]^{\frac{p-1}{p}}+\frac{(p G(d))^{\frac{p-1}{p}}}{(p-1) g(0)} .
\end{aligned}
$$

Letting $n \rightarrow+\infty$, we get a contradiction.

Let us denote by $u_{d} \in C(I)$ a fixed point of the mapping $T_{d}$ of Lemma 5 . 
Lemma 6. $\exists d>0$ such that $u_{d}(t) \geq 0 \quad \forall t \in\left[a, \frac{a+b}{2}[\right.$.

Proof. We know that $u_{d}$ is decreasing and that $u_{d}(a)=d$ for all $d>0$. Let us distinguish two cases. First if $\exists d>0$ such that $u_{d}(b) \geq 0$, then the conclusion of Lemma 6 clearly follows.

So we can assume that $\forall d>0: u_{d}(b)<0$. Since $\left.u_{d}(a)=d>0, \exists \delta_{d} \in\right] a, b[$ such that $u_{d}\left(\delta_{d}\right)=0$. It is clear that $u_{d}(t) \geq 0 \forall t \in\left[a, \delta_{d}[\right.$, and so it is sufficient to show that $\lim \sup _{d \rightarrow+\infty} \delta_{d}>\frac{a+b}{2}$.

Processing as in the proof of Lemma 5 we obtain

$$
(p-1)^{\frac{1}{p}}\left(-u_{d}^{\prime}(t)\right)\left\{p\left(G(d)-G\left(u_{d}(t)\right)\right)\right\}^{-\frac{1}{p}}=1 .
$$

Integrating from $a$ to $\delta_{d}$ and changing variable $s=u_{d}(t)$, one gets, $(p-1)^{\frac{1}{p}} \tau_{G}(d)=\delta_{d}-a$, and consequently

$$
\limsup _{d \rightarrow+\infty} \delta_{d}=a+(p-1)^{\frac{1}{p}} \limsup _{d \rightarrow+\infty} \tau_{G}(d) \text {. }
$$

Now one easily deduces from Lemma 4 that $\limsup _{d \rightarrow+\infty} \delta_{d}>a+\frac{b-a}{2}=\frac{a+b}{2}$.

Proof of Lemma 2 Continued. Denoting $u_{d}(t)$ by $u(t)$, we have $u \in C^{1}(I)$, $\Delta_{p} u \in C(I)$ and

$$
\left\{\begin{aligned}
-\Delta_{p} u(t) & =g(u(t)) & & \forall t \in I, \\
u(t) & \geq 0 & & \forall t \in\left[a, \frac{a+b}{2}\right], \\
u^{\prime}(a) & =0 . & &
\end{aligned}\right.
$$

Define a function $\beta_{1}$ from $[a, b]$ to $\mathbb{R}$ by

$$
\beta_{1}(t)=\left\{\begin{array}{lll}
u\left(\frac{3 a+b}{2}-t\right) & \text { if } & t \in\left[a, \frac{a+b}{2}\right], \\
u\left(t-\frac{b-a}{2}\right) & \text { if } \quad t \in\left[\frac{a+b}{2}, b\right] .
\end{array}\right.
$$

We will show that this function $\beta$ fulfills the conditions of Lemma 2 . To see this it is sufficient to show that:

(a) $\beta_{1}$ is nonegative in $[a, b]$,

(b) $\beta_{1} \in C^{1}([a, b])$,

(c) $\Delta_{p} \beta_{1} \in C([a, b])$ and $-\Delta_{p} \beta_{1}(t)=g\left(\beta_{1}(t)\right) \quad \forall t \in[a, b]$.

Proof of (a). If $a \leq t \leq \frac{a+b}{2}$, then $a \leq \frac{3 a+b}{2}-t \leq \frac{a+b}{2}$, and if $\frac{a+b}{2} \leq t \leq b$, then $a \leq t-\frac{b-a}{2} \leq \frac{a+b}{2}$, so that the conclusion follows from the sign of $u$ on $\left[a, \frac{a+b}{2}\right]$.

Proof of (b). $\beta_{1} \in C^{1}\left(\left[a, \frac{a+b}{2}[), \beta_{1} \in C^{1}(] \frac{a+b}{2}, b\right]\right)$, and moreover $\frac{d}{d t^{+}} \beta_{1}\left(\frac{a+b}{2}\right)=u^{\prime}(a)=0$ and $\frac{d}{d t^{-}} \beta_{1}\left(\frac{a+b}{2}\right)=u^{\prime}(a)=0$.

Proof of (c). We know that, $-\left(\left|u^{\prime}(t)\right|^{p-2} u^{\prime}(t)\right)^{\prime}=g(u(t))$ for $t \in[a, b]$ therefore

$$
-\left|u^{\prime}(t)\right|^{p-2} u^{\prime}(t)=\int_{a}^{t} g(u(s)) d s .
$$


If $\frac{a+b}{2} \leq t \leq b$ then $a \leq t-\frac{b-a}{2} \leq \frac{a+b}{2}$, which gives

$$
-\left(\left|u^{\prime}\left(t-\frac{b-a}{2}\right)\right|^{p-2} u^{\prime}\left(t-\frac{b-a}{2}\right)\right)=\int_{a}^{t-\frac{b-a}{2}} g(u(s)) d s .
$$

Changing variable $u=s+\frac{b-a}{2}$, this implies

$$
-\left|\beta_{1}^{\prime}(t)\right|^{p-2} \beta_{1}^{\prime}(t)=\int_{\frac{a+b}{2}}^{t} g\left(\beta_{1}(s)\right) d s,
$$

hence $-\Delta_{p} \beta_{1}(t)=g\left(\beta_{1}(t)\right)$ for all $t \in\left[\frac{a+b}{2}, b\right]$. The proof is similar for all $t \in\left[a, \frac{a+b}{2}\right]$.

\section{The CASE OF A MORE GENERAL OPERATOR.}

Let $\Omega$ be a bounded domain in $\mathbb{R}^{N}$ and let $A_{p}$ be an elliptic operator of the form

$$
A_{p}(u)=\sum_{1 \leq i, j \leq N} \frac{\partial}{\partial x_{i}}\left(|\nabla u|_{a}^{p-2} a_{i j}(x) \frac{\partial u}{\partial x_{j}}\right)
$$

where $\left(a_{i j}(x)\right)_{1 \leq i, j \leq N}$ are real-valued $L^{\infty}(\Omega)$

functions verifying $a_{i j}(x)=a_{j i}(x)$ for all $i, j$ and

(*) $\quad \sum_{1 \leq i, j \leq N} a_{i j}(x) \xi_{i} \xi_{j}=|\xi|_{a}^{2} \geq|\xi|^{2} \quad$ a.e. $x \in \Omega$ and for all $\xi \in \mathbb{R}^{N}$.

We now consider the problem

$$
\left(\mathcal{P}_{p}^{\prime}\right)\left\{\begin{array}{rlrl}
-A_{p} u & =f(u)+h & \text { in } \Omega, \\
u & =0 & & \text { on } \partial \Omega .
\end{array}\right.
$$

Note that $A_{p}$ is defined from $W_{0}^{1, p}(\Omega)$ to $W^{-1, p^{\prime}}(\Omega)$. Note also that $(*)$ implies that for each $i, a_{i, i}(x)>0$ a.e. in $\Omega$. We suppose that:

$$
\left\{\begin{array}{l}
\exists i^{\prime} \in\{1,2, \ldots, N\} \text { such that } a_{i^{\prime} i^{\prime}}=c t e \in \mathbb{R} \text { and } \\
\operatorname{div}\left(a_{1, i^{\prime}}(x), \ldots, a_{N, i^{\prime}}(x)\right)=\sum_{i \neq i^{\prime}} \frac{\partial}{\partial x_{i}} a_{i, i^{\prime}}(x)=0 .
\end{array}\right.
$$

We observe that $\left(A_{0}\right)$ holds in particular when $a_{i, i^{\prime}} i=1, \ldots, N$, are fixed constants.

Denote by $b=b_{i^{\prime}}$ and $a=a_{i^{\prime}}$ where $\left[a_{i^{\prime}}, b_{i^{\prime}}\right]$ is an edge of an arbitrary parallelepiped containing $\Omega$ such that $\left[a_{i^{\prime}}, b_{i^{\prime}}\right]$ is parallel to the $x_{i^{\prime}}$-axis and by

$$
C_{p}(b-a)=C_{p}=(p-1)\left\{\frac{2}{b-a} \int_{0}^{1} \frac{d t}{\left(1-t^{p}\right)^{\frac{1}{p}}}\right\}^{p}
$$

Theorem 2. Assume $\left(A_{0}\right)$ and

$$
\liminf _{s \rightarrow \pm \infty} \frac{p F(s)}{|s|^{p}}<\left(a_{i^{\prime} i^{\prime}}\right)^{\frac{p}{2}} C_{p} .
$$

Then $\left(\mathcal{P}^{\prime}\right)$ has a solution $u \in W_{0}^{1, p}(\Omega) \cap L^{\infty}(\Omega)$ for any $h \in L^{\infty}(\Omega)$.

The proof of Theorem 2 follows as in Theorem 1. Upper and lower solutions are defined for $A_{p}$ in the same way as in definition 1 relative to $\Delta_{p}$. 
Lemma 7. Assume that $\left(\mathcal{P}^{\prime}\right)$ admits an upper solution $\beta$ and a lower solution $\alpha$, then $\left(\mathcal{P}^{\prime}\right)$ admits a solution $u \in W_{0}^{1, p}(\Omega) \cap L^{\infty}(\Omega)$ with $\alpha(x) \leq u(x) \leq \beta(x)$.

The proof of Lemma 7 follows similar lines as Lemma 1. It sufficis to remark that $-A_{p}$ is strictly monotone.

Proof of Theorem 2. Let us describe the construction of the upper solution (that of lower solution is similar).

Let $g$ be the continuous function defined by

$$
g(s)=\frac{f(s)}{\left(a_{i^{\prime} i^{\prime}}\right)^{\frac{p}{2}}} \text { and denote } G(s)=\int_{0}^{s} g(t) d t .
$$

Then $\left(F_{2}\right)$ implies that $\liminf _{s \rightarrow \pm \infty} \frac{p G(s)}{|s|^{p}}<C_{p}$. By Lemma 2 with $M>\frac{\|h\|_{\infty}}{\left(a_{i^{\prime} i^{\prime}}\right)^{p-1}}$, there exists $\beta_{1} \in C^{1}(I)$ such that $\Delta_{p} \beta_{1} \in C(I)$ and

$$
\left\{\begin{aligned}
-\Delta_{p} \beta_{1}(t) & \geq g\left(\beta_{1}(t)\right)+M & \forall t \in I \\
\beta_{1}(t) & \geq 0 & \forall t \in I .
\end{aligned}\right.
$$

Writing $\beta(x)=\beta_{1}\left(x_{i}\right)$ for all $x \in \bar{\Omega}$, we have $\beta(x) \geq 0 \quad \forall x \in \bar{\Omega}, \beta \in C^{1}(\Omega)$. Morever, by $\left(A_{0}\right)$

$$
\begin{aligned}
A_{p}(u) & =\sum_{1 \leq i, j \leq N} \frac{\partial}{\partial x_{i}}\left(|\nabla \beta|_{a}^{p-2} a_{i j}(x) \frac{\partial \beta}{\partial x_{j}}\right) \\
& =\left(a_{i^{\prime} i^{\prime}}\right)^{\frac{p}{2}}\left(\left|\beta_{1}^{\prime}\left(x_{i^{\prime}}\right)\right|^{p-2} \beta_{1}^{\prime}\left(x_{i^{\prime}}\right)\right)^{\prime}+\left|\beta_{1}^{\prime}\left(x_{i^{\prime}}\right)\right|^{p-2} \beta_{1}^{\prime}\left(x_{i^{\prime}}\right) \sum_{i \neq i^{\prime}} \frac{\partial}{\partial x_{i}} a_{i i^{\prime}}(x) \\
& =\left(a_{i^{\prime} i^{\prime}}\right)^{\frac{p}{2}} \Delta_{p} \beta(x) .
\end{aligned}
$$

Hence

$A_{p} \beta \in C(\bar{\Omega})$ and $-A_{p} \beta(x)=-\left(a_{i^{\prime} i^{\prime}}\right)^{\frac{p}{2}} \Delta_{p} \beta(x) \geq f(\beta(x))+h(x)$ a.e.in $\Omega$, which shows that $\beta$ is an upper solution.

\section{Comments}

1. It is easy to give an example of a continuous function $f: \mathbb{R} \rightarrow \mathbb{R}$ such that

$$
\limsup _{ \pm \infty} \frac{p F(s)}{|s|^{p}}=+\infty \quad \text { and } \quad \liminf _{ \pm \infty} \frac{p F(s)}{|s|^{p}}=0
$$

(See the work of [Fe.O.Z] in the case $p=2$ ).

2. The problem $\left(\mathcal{P}_{p}\right)$ has at least one solution for any given $h \in L^{\infty}(\Omega)$ if we assume that:

$$
\limsup _{ \pm \infty} \frac{f(s)}{|s|^{p-2} s} \leq \lambda_{1}
$$

and

$$
\liminf _{ \pm \infty} \frac{p F(s)}{|s|^{p}}<\lambda_{1}
$$

This result was proved by Del Santo and Omari [S.O] for $p=2$, and was generalized by Elhachimi and Gossez [H,G.2] for $p>1$.

It is clear that $\left(f_{0}\right)$ is not verified in the example of comment 1 above. 
3. Positive density condition. Let $\eta>0$ and define $E=\left\{s \in \mathbb{R}^{*} ; \frac{p F(s)}{|s|^{p}}<C_{p}-\eta\right\}, \quad \tilde{E}=\left\{s \in \mathbb{R}^{*} ; \frac{p F(s)}{|s|^{p}}<\lambda_{1}-\eta\right\}$.

Theorem 3. (Defigueiredo and Gossez $[D, G]$ ) Assume

$$
\begin{gathered}
\exists a, b>0 \quad \text { such that }|f(s)| \leq a|s|^{p-1}+b \\
\limsup _{ \pm \infty} \frac{p F(s)}{|s|^{p} \leq \lambda_{1},} \\
\left\{\begin{array}{l}
\liminf _{r \rightarrow+\infty} \frac{\operatorname{meas}(\tilde{E} \cap[0, r])}{r}>0, \\
\liminf _{r \rightarrow-\infty} \frac{\operatorname{meas}(\tilde{E} \cap[r, 0])}{-r}>0 .
\end{array}\right.
\end{gathered}
$$

Then, for any $h \in W^{-1, p^{\prime}}(\Omega)$, there exists $u \in W_{0}^{1, p}(\Omega)$ solution of $\left(\mathcal{P}_{p}\right)$.

One says that $\tilde{E}$ has a positive density at $+\infty$ and $-\infty$ if (d) above is verified. This condition was introduced in $[\mathrm{D}, \mathrm{G}]$.

The question now naturally arises whether nonresonance still occurs in $\left(\mathcal{P}_{p}\right)$ when the "liminf" condition (d) is weakened into a "limsup" condition. We have:

Corollary to Theorem 1. Assume

$$
\left\{\begin{array}{l}
\limsup _{r \rightarrow+\infty} \frac{\text { meas }(E \cap[0, r])}{r}>0, \\
\limsup _{r \rightarrow-\infty} \frac{\operatorname{meas}(E \cap[r, 0])}{-r}>0 .
\end{array}\right.
$$

Then, for any $h \in L^{\infty}(\Omega)$, there exists $u \in W_{0}^{1, p}(\Omega)$

solution of $\left(\mathcal{P}_{p}\right)$.

Proof. Obviously $\left(d^{\prime}\right)$ implies that $E \cap \mathbb{R}_{-}$and $E \cap \mathbb{R}_{+}$are unbounded, so that $(F)$ is satisfied.

Remarks 1. (a) We have not supposed $\left(f_{0}\right)$ nor $\left(F_{0}\right)$ in the corollary. (b) The question whether we may assume only

$$
\left\{\begin{array}{l}
\limsup _{r \rightarrow+\infty} \frac{\operatorname{meas}(\tilde{E} \cap[0, r])}{r}>0, \\
\limsup _{r \rightarrow-\infty} \frac{\operatorname{meas}(\tilde{E} \cap[r, 0])}{-r}>0,
\end{array}\right.
$$

remains open. Note that the condition $\liminf _{s \rightarrow \pm \infty} \frac{p F(s)}{|s|^{p}}<\lambda_{1}$ is weaker than $(\tilde{d})$.

Acknowledgment. The authors wish to express their thanks to Professor Jean-Pierre Gossez for various interesting discussions on the subject of this paper. 


\section{REFERENCES}

[A] A. Anane, Simplicité et isolation de la première valeur propre du p-Laplacien avec poids, C. R. Acad. Sci. Paris Sr. I Math. 305 (1987), 725-728.

[B] E. di Benedetto, $C^{1+\alpha}$ local regularity of weak solutions of degenerate elliptic equations, Nonlinear Anal. 7 (1983), 827-850.

[D,G] D. G. de Figueiredo and J.-P.Gossez, Nonresonance below the first eigenvalue for a semilinear elliptic problem, Math. Ann. 281 (1988), 589-610.

[E,G.1] A. El Hachimi and J.-P. Gossez, A note on a nonresonance condition for a quasilinear elliptic problem, Nonlinear Anal. 22 (1994), p229-236.

[E,G.2] A. El Hachimi and J.-P.Gossez, On a nonresonance condition near the first eigenvalue for a quasilinear elliptic problem, Partial Differential Equations (Hansur-Lesse, 1993), 144-151, Math. Res., \#82, Akademie-Verlag, Berlin, 1994.

$[\mathrm{Fe}, \mathrm{O}, \mathrm{Z}] \quad$ M. Fernandes, P. Omari and F. Zanolin, On the solvability of a semilinear twopoint BVP around the first eigenvalue, Differential Integral Equations, 2 (1989), 63-79.

[F,G,Z] A. Fonda, J.-P.Gossez and F. Zanolin, On a nonresonance condition for a semilinear elliptic problem, Differential Integral Equations, 4 (1991), 945-951.

[GR,O.1] M. R. Grossinho and P. Omari, Solvabitity of the Dirichlet problem for a nonlinear parabolic equation under conditions on the potential, to appear.

[GR,O.2] M. R. Grossinho and P. Omari, A Hammerstein-type result for a semilinear parabolic problem, to appear.

[H] A. Hammerstein, Nichtlineare Integralgleichungen nebst Anwendungen, Acta Math. 54 (1930), 117-176.

[S,O] D. Del Santo and P.Omari, Nonresonance conditions on the potential for a semilinear elliptic problem, J. Differential Equations, 108 (1994), 120-138.

\section{DÉPARTEMENT DE MATHÉMATIQUES}

UNIVERSITÉ MOHAMED I

OUJDA, MOROCCO

E-mail addresses: anane@sciences.univ-oujda.ac.ma, chakrone@sciences.univ-oujda.ac.ma 


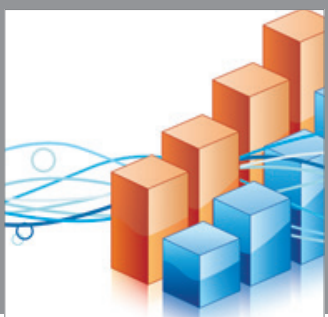

Advances in

Operations Research

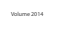

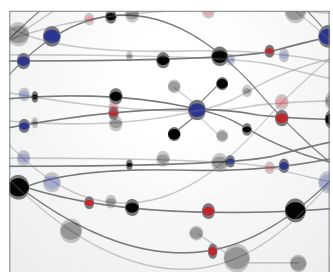

\section{The Scientific} World Journal
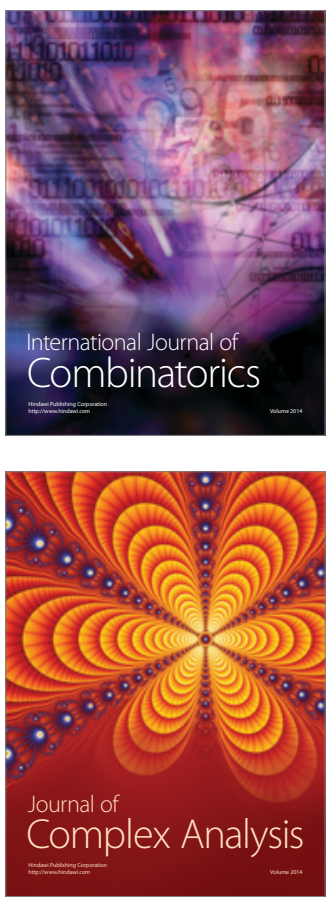

International Journal of

Mathematics and

Mathematical

Sciences
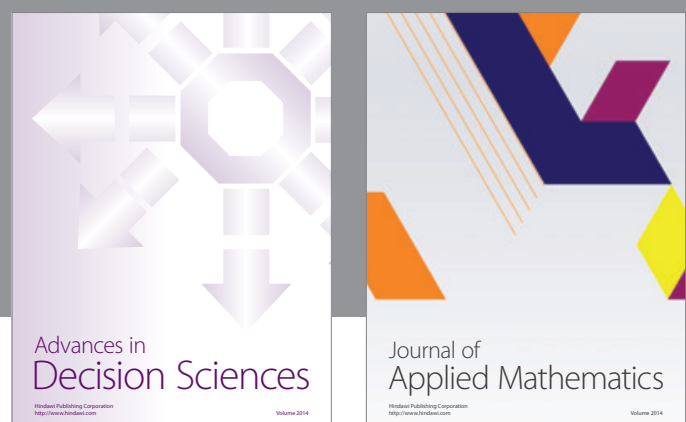

Journal of

Applied Mathematics
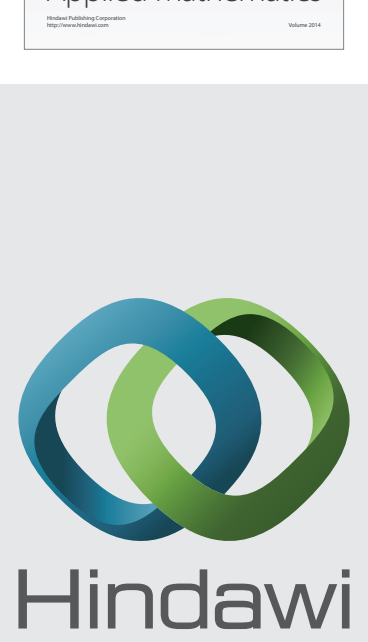

Submit your manuscripts at http://www.hindawi.com
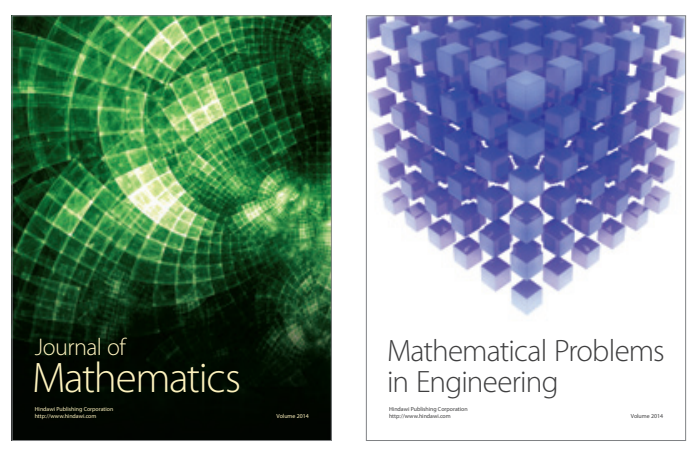

Mathematical Problems in Engineering
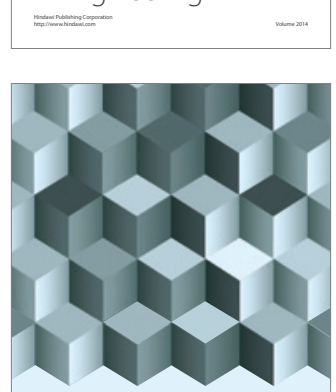

Journal of

Function Spaces
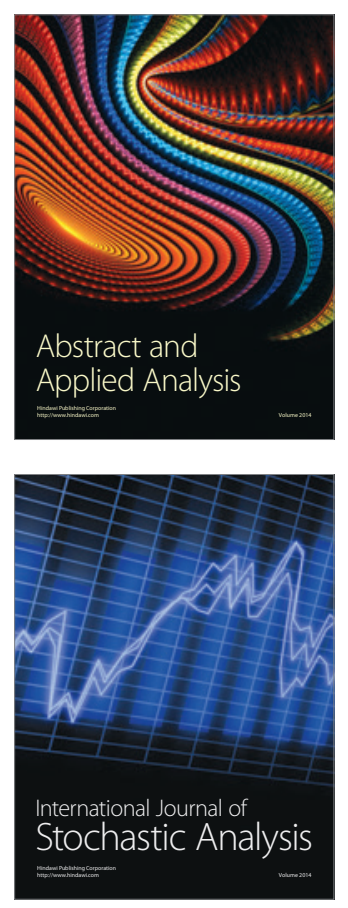

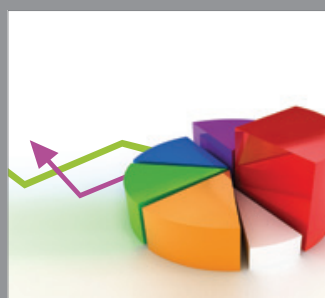

ournal of

Probability and Statistics

Promensencen
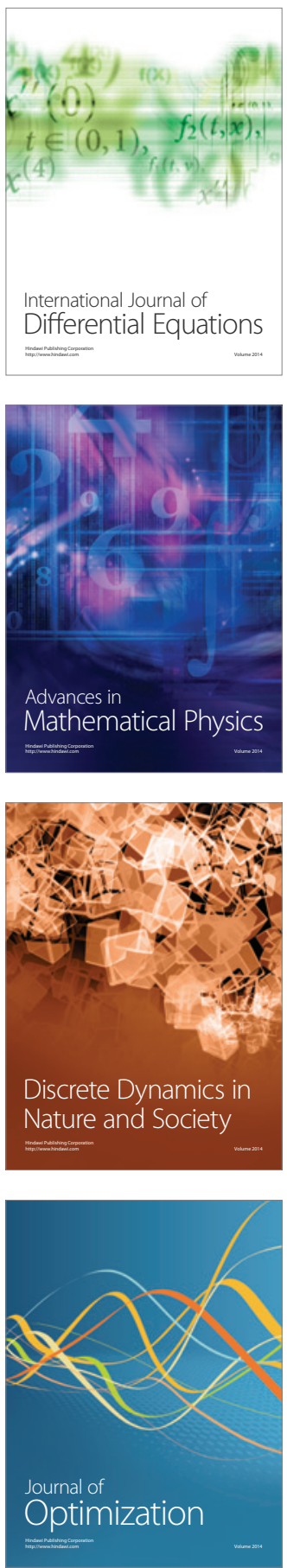